\title{
Reflection of quasi-P and quasi-SV waves at the free and rigid boundaries of a fibre-reinforced medium
}

\author{
A CHATTOPADHYAY, R L K VENKATESWARLU and S SAHA \\ Department of Applied Mathematics, Indian School of Mines, Dhanbad 826 004, \\ India \\ e-mail: c_amares@yahoo.com
}

MS received 25 May 2001; revised 28 August 2002

\begin{abstract}
The propagation of plane waves in fibre-reinforced media is discussed. The expressions of phase velocities of quasi-P (qP) and quasi-SV (qSV) waves propagating in plane symmetry are obtained in terms of propagation vectors. We have established a relation from which the displacement vector can be obtained in terms of the propagation vector. Expressions for the reflection coefficients of $\mathrm{qP}$ and qSV waves are obtained. Numerical results of reflection coefficients are obtained and presented graphically. The partition of energy between qP and qSV waves reflected on free and rigid boundaries due to incident qP and qSV waves are also obtained and presented graphically.
\end{abstract}

Keywords. Reflection of waves; quasi-P waves; quasi-SV waves; quasi-SH waves; fibre-reinforced media; reflection coefficients

\section{Introduction}

Fibre-reinforced composite materials have become very attractive in many engineering applications recently due to their superiority over other structural materials in applications requiring high strength and stiffness in lightweight components. Consequently the characterisation of their mechanical behaviour is of particular importance for structural design using these materials.

Effects of earthquakes on artificial structures are of prime importance to engineers and architects. During an earthquake and similar disturbances a structure is excited into a more or less violent vibration, with resulting oscillatory stresses, which depend both upon the ground vibration and physical properties of the structure (Richter 1958). Most concrete construction includes steel reinforcing, at least nominally. Thus wave propagation in a reinforced medium plays a very important role in civil engineering and geophysics.

The propagation of body waves in anisotropic media is fundamentally different from their propagation in isotropic media, although the differences may be comparatively subtle and difficult to observe (Crampin 1975). In general, for any type of anisotropy, there are always three types of body waves propagating with three different velocities. Choosing the three components of displacement adequately, they are called quasi-P(qP), quasi-SV(qSV) and quasi-SH 
(qSH) waves. The velocities of these three waves change according to the type of symmetry present in the medium. Owing to these properties, anisotropy is detected by observations of change in P-wave velocity along two perpendicular directions and by observations of S-wave splitting. For both these effects it is not necessary that the whole medium be anisotropic; only some part of it need be so (Udias 1999). Generally, particle motion is neither purely longitudinal nor purely transverse. For this reason, the three types of body waves in an anisotropic medium are referred as $\mathrm{qP}, \mathrm{qSV}$ and $\mathrm{qSH}$ instead of $\mathrm{P}, \mathrm{SV}$ and $\mathrm{SH}$.

The problem of reflection and refraction of elastic have been discussed by several authors. Without going into the details of the research work in this field, we mention the papers by Knott (1899), Gutenberg (1944), Thapliyal (1974), Keith \& Crampin (1977), Dey \& Addy (1979), Tolstoy (1982), Norris (1983), Pal \& Chattopadhyay (1984), Achenbach (1976), Henneke (1972), Chattopadhyay et al (1995) and Singh et al (2002), as giving a broad picture of the work done so far.

Crampin \& Taylor (1971) studied surface wave propagation in examples of unlayered and multi-layered anisotropic media which is examined numerically with a program using an extension of the Thompson-Haskell matrix formulation. They studied some examples of surface wave propagation in anisotropic media to interpret a possible geophysical structure. Crampin (1975) showed that the surface waves have distinct particle motion when propagating in a structure having a layer of anisotropic material with certain symmetry relations. Chattopadhyay \& Saha (1996) have studied the problem of reflection of qSV-wave at free and rigid boundary in a medium of monoclinic type.

The above mentioned authors have not studied the reflection behaviour at a free and rigid boundaries of a fibre-reinforced medium. The reflection of $\mathrm{qP}$ and $\mathrm{qSV}$ waves in a fibre-reinforced medium is discussed. In this paper we have computed the reflection coefficients of qP and qSV waves at the free and rigid boundary of a fibre-reinforced medium. It is well known that in an anisotropic medium the direction of particle motion is neither perpendicular nor parallel to the direction of propagation. Considering this fact, a relation has been established to calculate the displacement vector in terms of propagation vector. The expressions for phase velocity of $\mathrm{qP}$ and $\mathrm{qSV}$ waves are obtained in terms of the propagation vector. The partition of energy between $\mathrm{qP}$ and $\mathrm{qSV}$ waves reflected for $\mathrm{qP}$ and $\mathrm{qSV}$ waves incident on a free and rigid boundaries have been derived and presented graphically.

\section{Formulation of the problem}

The constitutive equations for fibre-reinforced linearly elastic medium whose preferred direction is that of a are (Spencer 1972)

$$
\begin{aligned}
\tau_{i j}= & \lambda e_{k k} \delta_{i j}+2 \mu_{T} e_{i j}+\alpha\left(a_{k} a_{m} e_{k m} \delta_{i j}+e_{k k} a_{i} a_{j}\right) \\
& +2\left(\mu_{L}-\mu_{T}\right)\left(a_{i} a_{k} e_{k j}+a_{j} a_{k} e_{k i}\right)+\beta\left(a_{k} a_{m} e_{k m} a_{i} a_{j}\right),
\end{aligned}
$$

where $\tau_{i j}$ are components of stress, $e_{i j}$ are components of infinitesimal strain, $a_{j}$ are components of $\mathbf{a}$, all referred to cartesian coordinates. The vector $\mathbf{a}$ may be a function of position. The coefficients $\lambda, \mu_{L}, \mu_{T}, \alpha$ and $\beta$ are elastic constants with the dimension of stress.

If $\mathbf{a}$ is so chosen that its components are $(1,0,0)$. The stress components (1) become

$$
\begin{aligned}
& \tau_{11}=\left(\lambda+2 \alpha+4 \mu_{L}-2 \mu_{T}+\beta\right) e_{11}+(\lambda+\alpha) e_{22}+(\lambda+\alpha) e_{33}, \\
& \tau_{22}=(\lambda+\alpha) e_{11}+\left(\lambda+2 \mu_{T}\right) e_{22}+\lambda e_{33},
\end{aligned}
$$




$$
\begin{aligned}
& \tau_{33}=(\lambda+\alpha) e_{11}+\lambda e_{22}+\left(\lambda+2 \mu_{T}\right) e_{33}, \\
& \tau_{12}=2 \mu_{L} e_{12}, \tau_{13}=2 \mu_{L} e_{13}, \tau_{23}=2 \mu_{T} e_{23},
\end{aligned}
$$

where $2 e_{i j}=u_{i, j}+u_{j, i}$ and $u_{i}(i=1,2,3)$ are the displacement components.

We take the plane of symmetry of the fibre-reinforced medium as the $x_{1} x_{2}$-plane and $x_{2}$ axis vertically upwards. For the plane wave propagation in $x_{1} x_{2}$-plane, we have $\partial / \partial x_{3}=0$.

The non-vanishing equations of motion without body forces are

$$
\begin{aligned}
& \frac{\partial \tau_{11}}{\partial x_{1}}+\frac{\partial \tau_{12}}{\partial x_{2}}=\rho \frac{\partial^{2} u_{1}}{\partial t^{2}}, \\
& \frac{\partial \tau_{21}}{\partial x_{1}}+\frac{\partial \tau_{22}}{\partial x_{2}}=\rho \frac{\partial^{2} u_{2}}{\partial t^{2}}, \\
& \frac{\partial \tau_{31}}{\partial x_{1}}+\frac{\partial \tau_{32}}{\partial x_{2}}=\rho \frac{\partial^{2} u_{3}}{\partial t^{2}},
\end{aligned}
$$

The stress equations of motion (3) with the help of (2) become

$$
\begin{aligned}
& \left(\lambda+2 \alpha+4 \mu_{L}-2 \mu_{T}+\beta\right) \frac{\partial^{2} u_{1}}{\partial x_{1}^{2}}+\mu_{L} \frac{\partial^{2} u_{1}}{\partial x_{2}^{2}}+\left(\lambda+\alpha+\mu_{L}\right) \frac{\partial^{2} u_{2}}{\partial x_{1} \partial x_{2}}=\rho \frac{\partial^{2} u_{1}}{\partial t^{2}} \\
& \mu_{L} \frac{\partial^{2} u_{2}}{\partial x_{1}^{2}}+\left(\lambda+2 \mu_{T}\right) \frac{\partial^{2} u_{2}}{\partial x_{2}^{2}}+\left(\lambda+\mu_{L}+\alpha\right) \frac{\partial^{2} u_{1}}{\partial x_{1} \partial x_{2}}=\rho \frac{\partial^{2} u_{2}}{\partial t^{2}} \\
& \mu_{L} \frac{\partial^{2} u_{3}}{\partial x_{1}^{2}}+\mu_{\mathrm{T}} \frac{\partial^{2} u_{3}}{\partial \mathrm{x}_{2}^{2}}=\rho \frac{\partial^{2} u_{3}}{\partial \mathrm{t}^{2}}
\end{aligned}
$$

From (4) to (6), it is obvious that qSH wave which is represented by $u_{3}$ motion in (6) is decoupled from $\left(u_{1}, u_{2}\right)$ motion representing $\mathrm{qP}$ and $\mathrm{qSV}$ waves. The phase velocity of qSH wave is

$$
\rho c_{n}^{2}=\mu_{L}\left\{p_{1}^{(n)}\right\}^{2}+\mu_{T}\left\{p_{2}^{(n)}\right\}^{2},
$$

where $\mathbf{p}\left(p_{1}^{(n)}, p_{2}^{(n)}, 0\right)$ denote the unit propagation vector, $c_{n}$ is the phase velocity and $k_{n}$ is the wave number of plane waves propagating in the $x_{1} x_{2}$-plane. We consider plane wave solutions of (4) and (5) as

$$
\left(\begin{array}{l}
u_{1} \\
u_{2}
\end{array}\right)=A\left(\begin{array}{l}
d_{1}^{(n)} \\
d_{2}^{(n)}
\end{array}\right) \exp \left[i k_{n}\left(\mathbf{x} \cdot \mathbf{p}-c_{n} t\right)\right],
$$

where $\mathbf{d}\left(d_{1}^{(n)}, d_{2}^{(n)}, 0\right)$ is the unit displacement vector.

Using the expressions of (8) for $u_{1}$ and $u_{2}$ in the equations of motion (4) and (5), we obtain

$$
\frac{d_{1}^{(n)}}{d_{2}^{(n)}}=\frac{S}{\rho c_{n}^{2}-R}=\frac{\rho c_{n}^{2}-T}{S},
$$

where

$$
\begin{aligned}
R & =\left(\lambda+2 \alpha+4 \mu_{L}-2 \mu_{T}+\beta\right)\left\{p_{1}^{(n)}\right\}^{2}+\mu_{L}\left\{p_{2}^{(n)}\right\}^{2}, \\
S & =\left(\lambda+\alpha+\mu_{L}\right) p_{1}^{(n)} p_{2}^{(n)}, \\
T & =\mu_{L}\left\{p_{1}^{(n)}\right\}^{2}+\left(\lambda+2 \mu_{T}\right)\left\{p_{2}^{(n)}\right\}^{2} .
\end{aligned}
$$


Equation (9) may be used to find the $\mathbf{d}$ in terms of $\mathbf{p}$.

From the above equation, we have

$$
\rho^{2} c_{n}^{4}-(R+T) \rho c_{n}^{2}+\left(R T-S^{2}\right)=0 .
$$

The solutions of the above equation are

$$
2 \rho c_{n}^{2}=(R+T) \pm\left[(R-T)^{2}+4 S^{2}\right]^{1 / 2} .
$$

Velocities of $\mathrm{qP}$ wave and $\mathrm{qSV}$ waves are

$$
\begin{aligned}
& 2 \rho c_{L}^{2}=(R+T)+\left((R-T)^{2}+4 S^{2}\right)^{1 / 2}, \\
& 2 \rho c_{T}^{2}=(R+T)-\left((R-T)^{2}+4 S^{2}\right)^{1 / 2},
\end{aligned}
$$

From (4) and (5), we obtain

$$
\begin{gathered}
{\left[\left(\lambda+2 \alpha+3 \mu_{L}-2 \mu_{T}+\beta\right) d_{1}^{(n)} d_{2}^{(n)}\left\{p_{1}^{(n)}\right\}^{2}\right]+\left(\mu_{L}-\lambda-2 \mu_{T}\right) d_{1}^{(n)} d_{2}^{(n)}\left\{p_{2}^{(n)}\right\}^{2}} \\
+\left(\lambda+\alpha+\mu_{L}\right)\left[\left\{d_{2}^{(n)}\right\}^{2}-\left\{d_{1}^{(n)}\right\}^{2}\right] p_{1}^{(n)} p_{2}^{(n)}=0
\end{gathered}
$$

Pure longitudinal and shear waves can propagate only in certain specific directions. Longitudinal and transverse specific directions are found by taking $\mathbf{d}=\mathbf{p}$ and $\mathbf{d}$ perpendicular to $\mathbf{p}$. In the anisotropic case no such relations can be considered between the displacement vector and the propagation vector.

We consider a homogeneous fibre-reinforced half-space occupying the region $x_{2} \leq 0$ and the plane of symmetry is taken as the $x_{1} x_{2}$-plane. Plane qP wave is incident at the tractionfree boundary $x_{2}=0$ and will generate reflected qP and qSV waves. Let $n=0,1,2$ be assumed for incident qP, reflected qP and reflected qSV waves respectively. We consider the plane strain problem and hence

$$
u_{1}=u_{1}\left(x_{1}, x_{2}, t\right), u_{2}=u_{2}\left(x_{1}, x_{2}, t\right), u_{3}=0 .
$$

The displacement field may be represented by

$$
u_{1}=\sum_{j=0}^{2} A_{j} d_{1}^{(j)} e^{i \eta_{j}}, u_{2}=\sum_{j=0}^{2} A_{j} d_{2}^{(j)} e^{i \eta_{j}},
$$

where

$$
\eta_{n}=k_{n}\left(x_{1} p_{1}^{(n)}+x_{2} p_{2}^{(n)}-c_{n} t\right) .
$$

For incident $\mathrm{qP}$ wave, which makes an angle $\theta_{0}$, we have

$$
p_{1}^{(0)}=\sin \theta_{0}, p_{2}^{(0)}=\cos \theta_{0}, c_{0}=c_{L} .
$$

In the plane $x_{2}=0$, the displacement and stress components due to incident qP-wave may be written as

$$
\begin{aligned}
& u_{1}^{(0)}=A_{0} d_{1}^{(0)} e^{i \eta_{0}}, u_{2}^{(0)}=A_{0} d_{2}^{(0)} e^{i \eta_{0}}, \\
& \tau_{22}^{(0)}=i A_{0} k_{0}\left[(\lambda+\alpha) d_{1}^{(0)} \sin \theta_{0}+\left(\lambda+2 \mu_{T}\right) d_{2}^{(0)} \cos \theta_{0}\right] e^{i \eta_{0}}, \\
& \tau_{21}^{(0)}=i A_{0} k_{0} \mu_{L}\left[d_{1}^{(0)} \cos \theta_{0}+d_{2}^{(0)} \sin \theta_{0}\right] e^{i \eta_{0}},
\end{aligned}
$$

where

$$
\eta_{0}=k_{0}\left(x_{1} p_{1}^{(0)}-c_{L} t\right)
$$


For a reflected qP wave which makes an angle $\theta_{1}$ we have

$$
p_{1}^{(1)}=\sin \theta_{1}, p_{2}^{(1)}=-\cos \theta_{1}, c_{1}=c_{L}^{\prime} .
$$

In the plane $x_{2}=0$, the displacement and stress components due to reflected qP-wave may be written as

$$
\begin{aligned}
& u_{1}^{(1)}=A_{1} d_{1}^{(1)} e^{i \eta_{1}}, u_{2}^{(1)}=A_{1} d_{2}^{(1)} e^{i \eta_{1}}, \\
& \tau_{22}^{(1)}=i A_{1} k_{1}\left[(\lambda+\alpha) d_{1}^{(1)} \sin \theta_{1}-\left(\lambda+2 \mu_{T}\right) d_{2}^{(1)} \cos \theta_{1}\right] e^{i \eta_{1}}, \\
& \tau_{21}^{(1)}=i A_{1} k_{1} \mu_{L}\left[-d_{1}^{(1)} \cos \theta_{1}+d_{2}^{(1)} \sin \theta_{1}\right] e^{i \eta_{1}},
\end{aligned}
$$

where

$$
\eta_{1}=k_{1}\left(x_{1} p_{1}^{(1)}-c_{L}^{\prime} t\right) .
$$

If the reflected qSV wave makes an angle $\theta_{2}$, we have

$$
p_{1}^{(2)}=\sin \theta_{2}, p_{2}^{(2)}=-\cos \theta_{2}, c_{2}=c_{T} .
$$

In the plane $x_{2}=0$, the displacement and stress components due to reflected qSV-wave may be written as

$$
\begin{aligned}
u_{1}^{(2)} & =A_{2} d_{1}^{(2)} e^{i \eta_{2}}, u_{2}^{(2)}=A_{2} d_{2}^{(2)} e^{i \eta_{2}}, \\
\tau_{22}^{(2)} & =i A_{2} k_{2}\left[(\lambda+\alpha) d_{1}^{(2)} \sin \theta_{2}-\left(\lambda+2 \mu_{T}\right) d_{2}^{(2)} \cos \theta_{2}\right] e^{i \eta_{2}}, \\
\tau_{21}^{(2)} & =i k_{2} A_{2} \mu_{L}\left[-d_{1}^{(2)} \cos \theta_{2}+d_{2}^{(2)} \sin \theta_{2}\right] e^{i \eta_{2}},
\end{aligned}
$$

where

$$
\eta_{2}=k_{2}\left(x_{1} p_{1}^{(2)}-c_{T} t\right)
$$

\section{Boundary conditions and solution of the problem for incident qP-waves}

Case 1: Reflection reflection of $\mathrm{qP}$-wave at a free boundary.

When $x_{2}=0$ is a free surface, the sum of the three tractions must vanish at $x_{2}=0$ and we can write the boundary conditions as:

$$
\tau_{22}^{(0)}+\tau_{22}^{(1)}+\tau_{22}^{(2)}=0,
$$

and

$$
\tau_{21}^{(0)}+\tau_{21}^{(1)}+\tau_{21}^{(2)}=0
$$

Substituting in (20), the values of $\tau_{22}^{(n)}, \tau_{21}^{(n)}$ (for $\left.n=0,1,2\right)$ from (16), (18) and (19), we obtain:

$$
\begin{aligned}
& i k_{0} A_{0}\left[(\lambda+\alpha) d_{1}^{(0)} \sin \theta_{0}+\left(\lambda+2 \mu_{T}\right) d_{2}^{(0)} \cos \theta_{0}\right] \exp \left(i \eta_{0}\right) \\
& \quad+i k_{1} A_{1}\left[(\lambda+\alpha) d_{1}^{(1)} \sin \theta_{1}-\left(\lambda+2 \mu_{T}\right) d_{2}^{(1)} \cos \theta_{1}\right] \exp \left(i \eta_{1}\right) \\
& \quad+i k_{2} A_{2}\left[(\lambda+\alpha) d_{1}^{(2)} \sin \theta_{2}-\left(\lambda+2 \mu_{T}\right) d_{2}^{(2)} \cos \theta_{2}\right] \exp \left(i \eta_{2}\right)=0
\end{aligned}
$$


and

$$
\begin{aligned}
& i k_{0} A_{0}\left[d_{1}^{(0)} \cos \theta_{0}+d_{2}^{(0)} \sin \theta_{0}\right] \exp \left(i \eta_{0}\right) \\
& \quad+i k_{1} A_{1}\left[-d_{1}^{(1)} \cos \theta_{1}+d_{2}^{(1)} \sin \theta_{1}\right] \exp \left(i \eta_{1}\right) \\
& \quad+i k_{2} A_{2}\left[-d_{1}^{(2)} \cos \theta_{2}+d_{2}^{(2)} \sin \theta_{2}\right] \exp \left(i \eta_{2}\right)=0 .
\end{aligned}
$$

Equations (21) and (22) must be valid for all values of $x_{1}$ and $t$, hence

$$
\eta_{0}=\eta_{1}=\eta_{2}
$$

which means

$$
k_{0}\left(x_{1} \sin \theta_{0}-c_{L} t\right)=k_{1}\left(x_{1} \sin \theta_{1}-c_{L}^{\prime} t\right)=k_{2}\left(x_{1} \sin \theta_{2}-c_{T} t\right) .
$$

This gives

$$
k_{0} \sin \theta_{0}=k_{1} \sin \theta_{1}=k_{2} \sin \theta_{2}=\phi
$$

and

$$
k_{0} c_{L}=k_{1} c_{L}^{\prime}=k_{2} c_{T}=\omega,
$$

where $\phi$ is the apparent wave number, and $\omega$ is the circular frequency.

From the above relations we have

$$
\frac{k_{1}}{k_{0}}=\frac{c_{L}}{c_{L}^{\prime}}=\frac{\sin \theta_{0}}{\sin \theta_{1}}
$$

and

$$
\frac{k_{2}}{k_{0}}=\frac{c_{L}}{c_{T}}=\frac{\sin \theta_{0}}{\sin \theta_{2}} .
$$

Equations (21) and (22) after using (23) may be written as

$$
\begin{aligned}
& P_{0} A_{0}+P_{1} A_{1}+P_{2} A_{2}=0, \\
& P_{3} A_{0}+P_{4} A_{1}+P_{5} A_{2}=0,
\end{aligned}
$$

where

$$
\begin{aligned}
& P_{0}=k_{0}\left[(\lambda+\alpha) d_{1}^{(0)} \sin \theta_{0}+\left(\lambda+2 \mu_{\mathrm{T}}\right) d_{2}^{(0)} \cos \theta_{0}\right], \\
& P_{1}=k_{1}\left[(\lambda+\alpha) d_{1}^{(1)} \sin \theta_{1}-\left(\lambda+2 \mu_{\mathrm{T}}\right) d_{2}^{(1)} \cos \theta_{1}\right], \\
& P_{2}=k_{2}\left[(\lambda+\alpha) d_{1}^{(2)} \sin \theta_{2}-\left(\lambda+2 \mu_{\mathrm{T}}\right) d_{2}^{(2)} \cos \theta_{2}\right], \\
& P_{3}=k_{0}\left[d_{1}^{(0)} \cos \theta_{0}+d_{2}^{(0)} \sin \theta_{0}\right], \\
& P_{4}=k_{1}\left[-d_{1}^{(1)} \cos \theta_{1}+d_{2}^{(1)} \sin \theta_{1}\right], \\
& P_{5}=k_{2}\left[-d_{1}^{(2)} \cos \theta_{2}+d_{2}^{(2)} \sin \theta_{2}\right] .
\end{aligned}
$$

Solving the above two equations, we have,

$$
\frac{A_{1}}{A_{0}}=\frac{a_{2}-b_{2}}{a_{1} b_{2}-a_{2} b_{1}}, \frac{A_{2}}{A_{0}}=-\frac{a_{1}-b_{1}}{a_{1} b_{2}-a_{2} b_{1}},
$$


where

$$
a_{1}=\frac{P_{1}}{P_{0}}, a_{2}=\frac{P_{2}}{P_{0}}, b_{1}=\frac{P_{4}}{P_{3}}, b_{2}=\frac{P_{5}}{P_{3}} .
$$

$d_{1}^{(i)} / d_{2}^{(i)}(i=0,1,2)$ can be calculated from (9) and are as under:

$$
d_{1}^{(0)} / d_{2}^{(0)}=\left(\rho c_{L}^{2}-T\right) / S
$$

where $\mathrm{R}, \mathrm{S}$ and $\mathrm{T}$ can be calculated after putting $p_{1}=p_{1}^{(0)}=\sin \theta_{0}$ and $p_{2}=p_{2}^{(0)}=\cos \theta_{0}$ in (10).

$$
d_{1}^{(1)} / d_{2}^{(1)}=\left(\rho c_{L}^{\prime 2}-T_{1}\right) / S_{1}
$$

where $R_{1}, S_{1}$ and $T_{1}$ can be calculated after putting $p_{1}=p_{1}^{(1)}=\sin \theta_{1}$ and $p_{2}=p_{2}^{(1)}=$ $-\cos \theta_{1}$ in (10).

$$
d_{1}^{(2)} / d_{2}^{(2)}=\left(\rho c_{T}^{2}-T_{2}\right) / S_{2}
$$

where $R_{2}, S_{2}$ and $T_{2}$ can be calculated after putting $p_{1}=p_{1}^{(2)}=\sin \theta_{2}$ and $p_{2}=p_{2}^{(2)}=$ $-\cos \theta_{2}$ in (10).

From (11) and (12), the velocities of incident $\mathrm{qP}$, reflected $\mathrm{qP}$ and reflected $\mathrm{qSV}$ may be defined by

$$
\begin{aligned}
2 \rho c_{L}^{2} & =(R+T)+\left((R-T)^{2}+4 S^{2}\right)^{1 / 2}, \\
2 \rho c^{\prime 2} & =\left(R_{1}+T_{1}\right)+\left(\left(R_{1}-T_{1}\right)^{2}+4 S_{1}^{2}\right)^{1 / 2}, \\
2 \rho c_{T}^{2} & =\left(R_{2}+T_{2}\right)-\left(\left(R_{2}-T_{2}\right)^{2}+4 S_{2}^{2}\right)^{1 / 2},
\end{aligned}
$$

where $R, R_{1}, R_{2}, S, S_{1}, S_{2}, T, T_{1}$ and $T_{2}$ are defined in (29) to (31).

Using the following values of reinforced-free medium

$$
\mu_{L}=\mu_{T}=\mu, \alpha=\beta=0,
$$

equations (28) reduce to

$$
\begin{aligned}
& \frac{A_{1}}{A_{0}}=\frac{\sin 2 \theta_{0} \sin 2 \theta_{2}-\bar{K}^{2} \cos ^{2} 2 \theta_{2}}{\sin 2 \theta_{0} \sin 2 \theta_{2}+\bar{K}^{2} \cos ^{2} 2 \theta_{2}} \\
& \frac{A_{2}}{A_{0}}=\frac{2 \bar{K} \sin 2 \theta_{0} \cos 2 \theta_{2}}{\sin 2 \theta_{0} \sin 2 \theta_{2}+\bar{K}^{2} \cos ^{2} 2 \theta_{2}}
\end{aligned}
$$

where,

$$
\bar{K}=[(\lambda+2 \mu) / \mu]^{1 / 2}
$$

which are the reflection coefficients of $\mathrm{P}$ and $\mathrm{SV}$ waves respectively for free boundary in isotropic case (Achenbach 1976, p. 175). by

The partition of energy between reflected $\mathrm{qP}$ and $\mathrm{qSV}$ waves for incident $\mathrm{qP}$ wave is given

$$
\left(\frac{A_{1}}{A_{0}}\right)^{2} \frac{c_{L}}{c_{L}^{l}} \frac{m_{1} \cos \theta_{1}}{m_{0} \cos \theta_{0}}+\left(\frac{A_{2}}{A_{0}}\right)^{2} \frac{c_{L}}{c_{T}} \frac{m_{2} \cos \theta_{2}}{m_{0} \cos \theta_{0}}=1
$$


where

$$
\begin{aligned}
m_{0}= & \left(a p_{1}^{(0)} d_{1}^{(0)}+b p_{2}^{(0)} d_{2}^{(0)}\right) \sin \theta_{0} d_{1}^{(0)}+\mu_{L}\left(d_{1}^{(0)} p_{2}^{(0)}+d_{2}^{(0)} p_{1}^{(0)}\right) \\
& \times\left(d_{1}^{(0)} \cos \theta_{0}+d_{2}^{(0)} \sin \theta_{0}\right)+\left(b d_{1}^{(0)} p_{1}^{(0)}+c d_{2}^{(0)} p_{2}^{(0)}\right) d_{2}^{(0)} \cos \theta_{0}, \\
m_{1}= & \left(a p_{1}^{(1)} d_{1}^{(1)}+b p_{2}^{(1)} d_{2}^{(1)}\right) \sin \theta_{1} d_{1}^{(1)}+\mu_{L}\left(d_{1}^{(1)} p_{2}^{(1)}+d_{2}^{(1)} p_{1}^{(1)}\right) \\
& \times\left(-d_{1}^{(1)} \cos \theta_{1}+d_{2}^{(1)} \sin \theta_{1}\right)-\left(b p_{1}^{(1)} d_{1}^{(1)}+c p_{2}^{(1)} d_{2}^{(1)}\right) d_{2}^{(1)} \cos \theta_{1}, \\
m_{2}= & \left(a p_{1}^{(2)} d_{1}^{(2)}+b p_{2}^{(2)} d_{2}^{(2)}\right) \sin \theta_{2} d_{1}^{(2)}+\mu_{L}\left(d_{1}^{(2)} p_{2}^{(2)}+d_{2}^{(2)} p_{1}^{(2)}\right) \\
& \times\left(-d_{1}^{(2)} \cos \theta_{2}+d_{2}^{(2)} \sin \theta_{2}\right)-\left(b p_{1}^{(2)} d_{1}^{(2)}+c p_{2}^{(2)} d_{2}^{(2)}\right) d_{2}^{(2)} \cos \theta_{2} . \\
a= & \lambda+2 \alpha+4 \mu_{L}-2 \mu_{T}+\beta, b=\lambda+\alpha, c=\lambda+2 \mu_{T} .
\end{aligned}
$$

For an isotropic case, (35) becomes

$$
\left(\frac{A_{1}}{A_{0}}\right)^{2}+\left(\frac{A_{2}}{A_{0}}\right)^{2} \frac{c_{T}}{c_{L}} \frac{\cos \theta_{2}}{\cos \theta_{0}}=1,
$$

which is same as that of Achenbach (1976, p. 182).

The velocity of surface wave can be obtained from (28) by equating the denominator to zero. It has been observed that the surface wave velocity at $\theta=8.8^{\circ}$ in the case of a fibrereinforced medium is 1.58 times more than the Rayleigh wave in the classical case (values of $\lambda, \alpha, \beta, \mu_{T}$ and $\mu_{L}$ are defined in (6).

Case 2: Reflection of $\mathrm{qP}$-wave at a rigid boundary.

Since the boundary $x_{2}=0$ is bounded by a rigid layer, the boundary conditions may be taken as

$$
u_{1}^{(0)}+u_{1}^{(1)}+u_{1}^{(2)}=0,
$$

and

$$
u_{2}^{(0)}+u_{2}^{(1)}+u_{2}^{(2)}=0 .
$$

Substituting the values of $u_{1}^{(n)}, u_{2}^{(n)}$ for $n=0,1,2$ from (16), (18) and (19) in (36), we get

$$
\begin{aligned}
& A_{0} d_{1}^{(0)} \exp \left(i \eta_{0}\right)+A_{1} d_{1}^{(1)} \exp \left(i \eta_{1}\right)+A_{2} d_{1}^{(2)} \exp \left(i \eta_{2}\right)=0 \\
& A_{0} d_{2}^{(0)} \exp \left(i \eta_{0}\right)+A_{1} d_{2}^{(1)} \exp \left(i \eta_{1}\right)+A_{2} d_{2}^{(2)} \exp \left(i \eta_{2}\right)=0 .
\end{aligned}
$$

Solving the above two equations, we have

$$
\begin{aligned}
& \frac{A_{1}}{A_{0}}=\frac{d_{1}^{(2)} d_{2}^{(0)}-d_{2}^{(2)} d_{1}^{(0)}}{d_{1}^{(1)} d_{2}^{(2)}-d_{2}^{(1)} d_{1}^{(2)}} \\
& \frac{A_{2}}{A_{0}}=\frac{d_{1}^{(0)} d_{2}^{(1)}-d_{1}^{(1)} d_{2}^{(0)}}{d_{1}^{(1)} d_{2}^{(2)}-d_{2}^{(1)} d_{1}^{(2)}} .
\end{aligned}
$$

The above equations are the reflection coefficients of $\mathrm{qP}$ and $\mathrm{qSV}$ waves for rigid boundaries. 


\section{Reflection of qSV waves at a free boundary}

Incident qSV wave will generate reflected qP and qSV waves. Let $n=0,1,2$ be assumed for incident qSV, reflected qP and reflected qSV waves respectively. For incident qSV wave, which makes an angle $\theta_{0}$, we have

$$
p_{1}^{(0)}=\sin \theta_{0}, p_{2}^{(0)}=\cos \theta_{0}, c_{0}=c^{\prime}{ }_{T} .
$$

In the plane $x_{2}=0$, the displacement and stress components of incident wave and reflected waves are same as in (16), (18) and (19). Equation (17) to be replaced by

$$
\eta_{0}=k_{0}\left(x_{1} p_{1}^{(0)}-c^{\prime}{ }_{T} t\right)
$$

\section{Boundary conditions and solution of the problem for $\mathrm{qSV}$ waves}

\subsection{Reflection of $q S V$ wave at a free boundary}

Substituting in (20), the values of $\tau_{22}^{(n)}, \tau_{21}^{(n)}$ (for $\left.n=0,1,2\right)$ from (41), (18) and (19), we obtain the same expressions as (21) and (22) except that $c_{L}$ is replaced by $c^{\prime}{ }_{T}$. The ratio $d_{1}^{(0)} / d_{2}^{(0)}$ is mentioned in (43), other ratios are the same as (30) and (31).

Equations (21) and (22) must be valid for all values of $x_{1}$ and $t$, hence

$$
\begin{aligned}
k_{0} \sin \theta_{0} & =k_{1} \sin \theta_{1}=k_{2} \sin \theta_{2}=\phi, \\
\text { and } \quad k_{0} c^{\prime}{ }^{\prime} & =k_{1} c^{\prime}{ }^{\prime}=k_{2} c_{T}=\omega,
\end{aligned}
$$

where $\phi$ and $\omega$ are defined in (24). Solving (21) and (22) we have the same sets of equations as (26) with some changes as mentioned below:

$d_{1}^{(i)} / d_{2}^{(i)}(i=0,1,2)$, may be calculated from (9) and are as under.

$$
d_{1}^{(0)} / d_{2}^{(0)}=\left(\rho c_{T}^{\prime 2}-T\right) / S
$$

where R, S and T can be calculated after taking $p_{1}=p_{1}^{0}=\sin \theta_{0}$ and $p_{2}=p_{2}^{(0)}=\cos \theta_{0}$ in (10). $d_{1}^{(i)} / d_{2}^{(i)}(i=1,2)$ are defined in (30) and (31).

From (12) and (11), the velocity of incident qSV may be defined by

$$
2 \rho c_{T}^{\prime 2}=(R+S)-\left((R-S)^{2}+4 T^{2}\right)^{1 / 2} .
$$

Reflected qP and qSV waves velocities are already defined in (32).

Using the following values of reinforced- free medium (values are mentioned in section 3 ), we obtain the reflection coefficients for isotropic case as

$$
\begin{aligned}
& \frac{A_{1}}{A_{0}}=-\frac{\bar{K} \sin 4 \theta_{0}}{\sin 2 \theta_{0} \sin 2 \theta_{1}+\bar{K}^{2} \cos ^{2} 2 \theta_{0}}, \\
& \frac{A_{2}}{A_{0}}=\frac{\sin 2 \theta_{0} \sin 2 \theta_{1}-\bar{K}^{2} \cos ^{2} 2 \theta_{0}}{\sin 2 \theta_{0} \sin 2 \theta_{1}+\bar{K}^{2} \cos ^{2} 2 \theta_{0}},
\end{aligned}
$$

where $\bar{K}=[(\lambda+2 \mu) / \mu]^{1 / 2}$. 
The partition of energy between reflected $\mathrm{qP}$ and $\mathrm{qSV}$ waves due to incident $\mathrm{qSV}$ wave is given by

$$
\left(\frac{A_{1}}{A_{0}}\right)^{2} \frac{c^{\prime}{ }_{T}}{c_{L}^{\prime}} \frac{m_{1} \cos \theta_{1}}{m_{0} \cos \theta_{0}}+\left(\frac{A_{2}}{A_{0}}\right)^{2} \frac{c^{\prime}{ }_{T}}{c_{T}} \frac{m_{2} \cos \theta_{2}}{m_{0} \cos \theta_{0}}=1
$$

where all definitions are as in (35).

\subsection{Reflection of $q S V$ wave at a rigid boundary}

Since the boundary $x_{2}=0$ is a rigid layer, the boundary conditions are the same as for (36).

After substituting the values of $u_{1}^{(n)}, u_{2}^{(n)}$ for $n=0,1,2$ from (41), (18) and (19) in (36), and solving, we have the same sets of equations as in (39) for reflection coefficients of $\mathrm{qP}$ and qSV waves.

Here $d_{1}^{(i)} / d_{2}^{(i)}(i=0,1,2)$ are as defined in (43), (30) and (31).

\section{Numerical calculations and discussions}

The material constants for fibre-reinforced medium have been considered as per Markham (1970).

$$
\begin{aligned}
\mu_{T} & =2.46 \times 10^{9} \mathrm{~N} / \mathrm{m}^{2}, \mu_{L}=5.66 \times 10^{9} \mathrm{~N} / \mathrm{m}^{2}, \\
\lambda & =5.65 \times 10^{9} \mathrm{~N} / \mathrm{m}^{2}, \beta=220.90 \times 10^{9} \mathrm{~N} / \mathrm{m}^{2}, \\
\alpha & =-1.28 \times 10^{9} \mathrm{~N} / \mathrm{m}^{2}, \rho=7800 \mathrm{~kg} / \mathrm{m}^{3} .
\end{aligned}
$$

\subsection{Reflection of $q P$ waves}

In figure 1, curve II corresponds to reflection coefficient of $\mathrm{qP}$-wave in fibre-reinforced medium. All the values of curve II are negative except from $0^{\circ}$ to $10^{\circ}$ and from $83^{\circ}$ to $90^{\circ}$. In

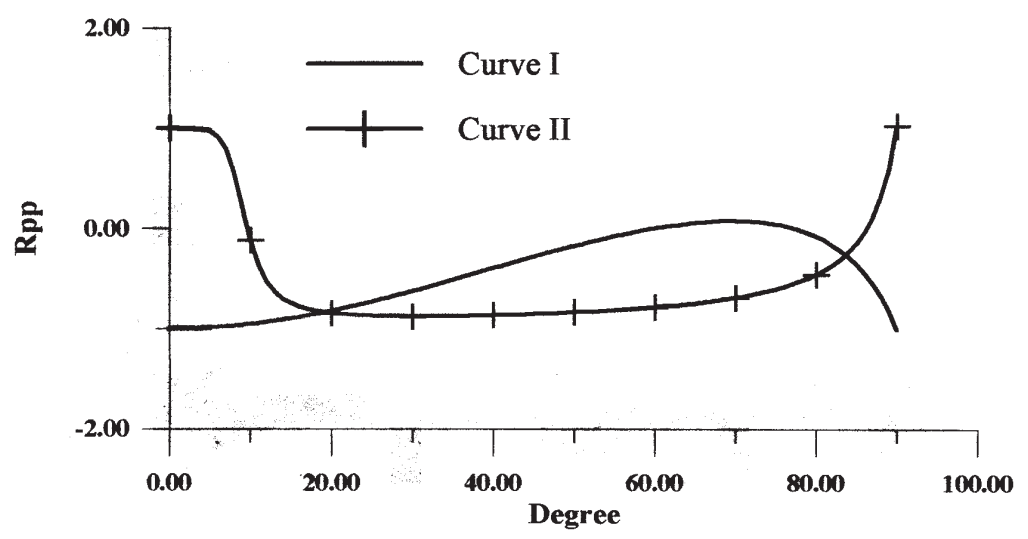

Figure 1. Amplitude ratios of $\mathrm{qP}$ waves due to incident $\mathrm{qP}$ waves. 


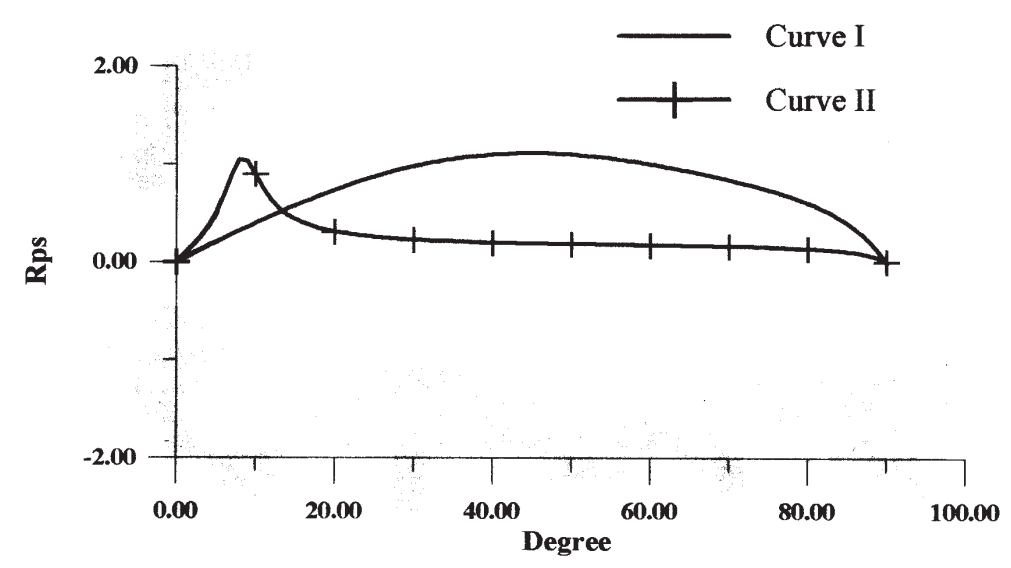

Figure 2. Amplitude ratios of $\mathrm{qSV}$ waves due to incident $\mathrm{qP}$ waves.

the isotropic case (curve I), the values of $\left(A_{1} / A_{0}\right)$ are all negative except from $\theta_{0}=57^{\circ}$ to $78^{\circ}$. Significant differences of values exist from $0^{\circ}$ to $5^{\circ}$ and from $80^{\circ}$ to $90^{\circ}$ in fibre-reinforced case compared to isotropic case. The values from $21^{\circ}$ to $80^{\circ}$ are greater in the isotropic case compared to those in the fibre-reinforced case.

In figure 2, the reflection coefficients of qSV waves for a free boundary of fibre-reinforced medium at different angles of incidence have plotted along with the curve for isotropic medium. The values of $\left(A_{2} / A_{0}\right)$ are all positive and equal for curves I and II at $\theta_{0}=0^{\circ}, 15^{\circ}$ and $90^{\circ}$. The difference in values at $\theta_{0}=50^{\circ}$ in isotropic case is significantly more compared to fibre-reinforced medium.

Figure 3 shows the comparison of partition of energy between reflected $\mathrm{P}$ and $\mathrm{qP}$ waves for incident $\mathrm{qP}$ waves. In this case $A_{1} / A_{0}=0$, for angle of incidence at $60^{\circ}$ and $78^{\circ}$, and $A_{1} / A_{0}=1$ for angle of incidence at $0^{\circ}$ and $90^{\circ}$ in case of isotropic media. For fibre-reinforced medium (curve II), $A_{1} / A_{0}=1$ at $0^{\circ}$ only.

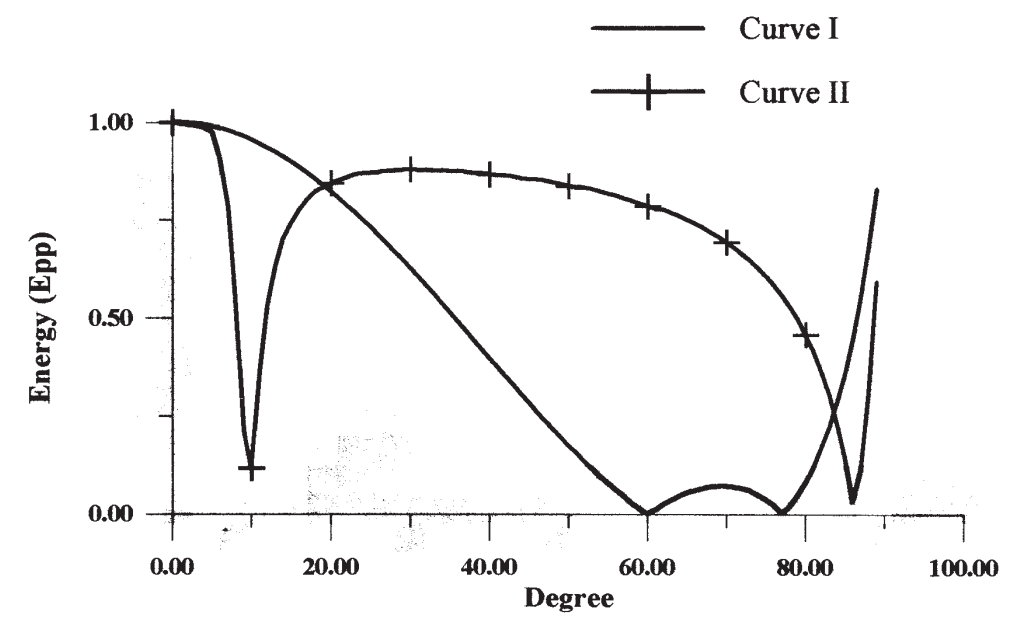

Figure 3. Partition of energy of $\mathrm{qP}$ waves due to incident $\mathrm{qP}$ waves. 


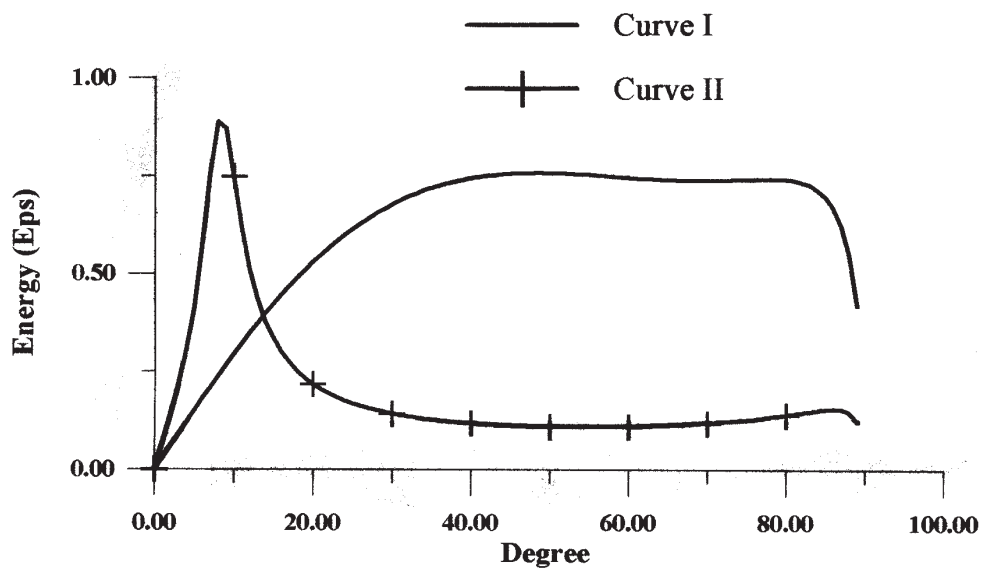

Figure 4. Partition of energy of $\mathrm{qSV}$ waves due to incident $\mathrm{qP}$ waves.

Figure 4 shows the comparison of partition of energy between reflected SV and qSV waves for incident qP waves. In this case $A_{2} / A_{0}=0$ at $0^{\circ}$ for both isotropic and fibre-reinforced (curve II) media. The critical point exists at $13^{\circ}$.

In figure 5, the values of reflection coefficients of qP-wave for isotropic (curve I) and fibre-reinforced media (curve II) have been plotted for rigid boundaries. The values of $A_{1} / A_{0}$ for fibre-reinforced media sharply increase from $0^{\circ}$ to $18^{\circ}$ and then remain constant from $19^{\circ}$ to $90^{\circ}$. The value of $A_{1} / A_{0}$ at $90^{\circ}$ is significantly more in case of a fibre-reinforced medium compared to the isotropic case, and at $0^{\circ}$ the value in the isotropic case is more compared to that in the fibre-reinforced case. The critical point exists at $18^{\circ}$.

In figure 6 , the values of reflection coefficient of qSV $\left(A_{2} / A_{0}\right)$ have been plotted for rigid boundary in curves II and I. It has been observed that all the values of the reflection

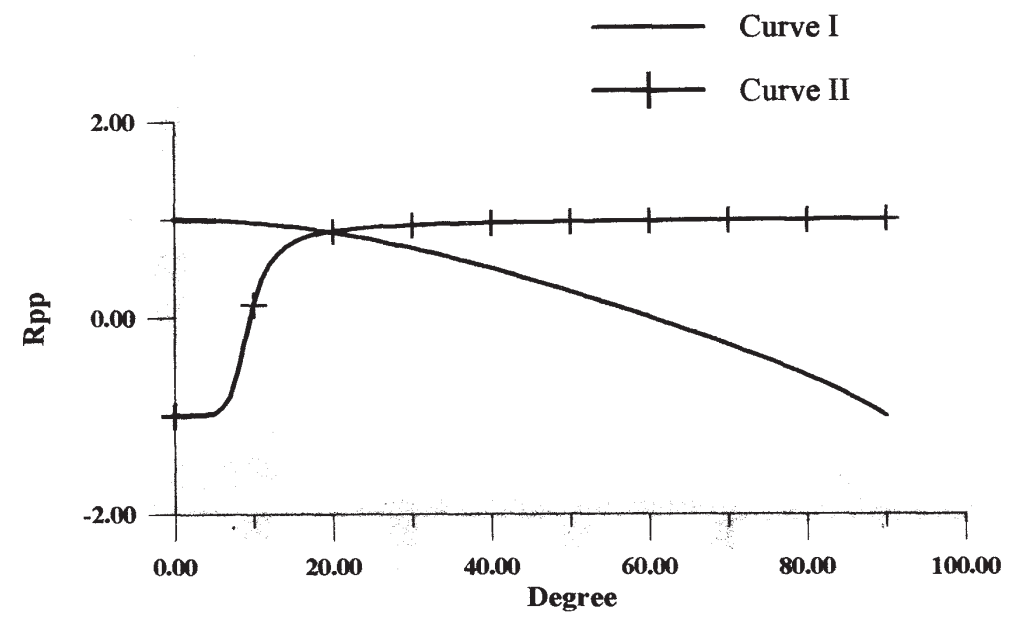

Figure 5. Amplitude ratios of $\mathrm{qP}$ waves due to incident $\mathrm{qP}$ waves in rigid boundary. 


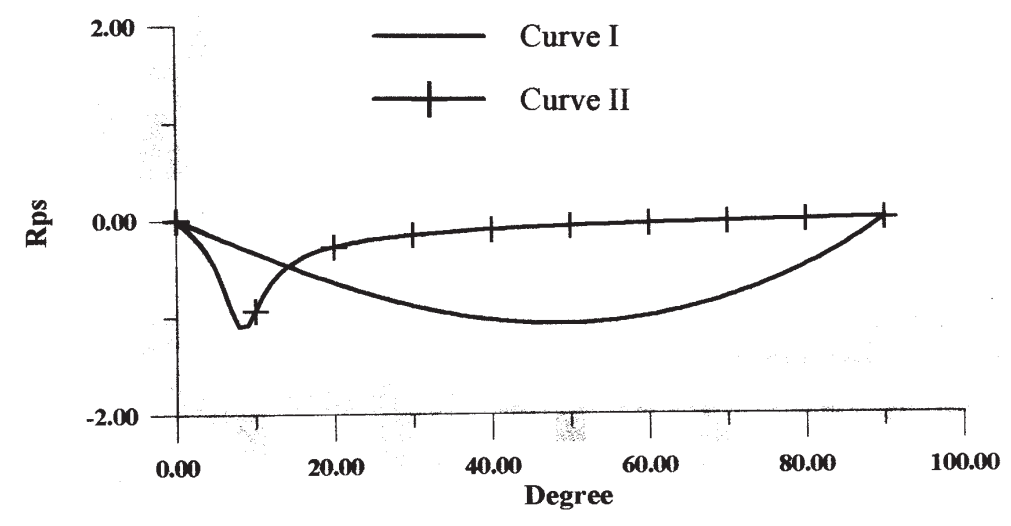

Figure 6. Amplitude ratios of qSV waves due to incident qP waves in rigid boundary.

coefficients of qSV are negative except those at $0^{\circ}$ and $90^{\circ}$. The values are greater in case of fibre-reinforced media as compared to isotropic media from $15^{\circ}$ to $89^{\circ}$. The maximum difference exists at $10^{\circ}$. The critical point exists at $15^{\circ}$.

Figures 7 and 8 show the partition of energy between reflected $\mathrm{qP}$ and $\mathrm{qSV}$ waves for incidence of a $\mathrm{qP}$ wave in a rigid boundary.

\subsection{Reflection of $q S V$ waves}

In figure 9, curve I corresponds to isotropic medium and agrees with the result of Achenbach (1976). Curve II corresponds to fibre-reinforced medium. The value of $A_{1} / A_{0}$ in a fibrereinforced medium is greater compared to that in isotropic medium but the difference is greater at $33^{\circ}$.

In figure 10, the reflection coefficient of qSV-waves (curve II) for reinforced medium for different values of $\theta_{0}$ ranging from $0^{\circ}$ to $33^{\circ}$ have been plotted which are permissible of $\theta_{0}$

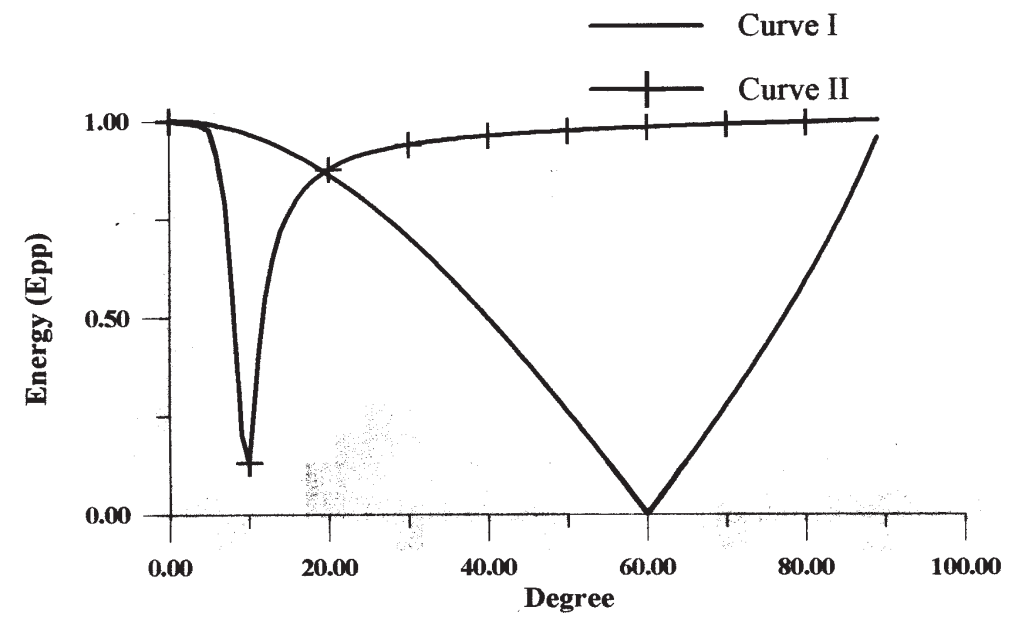

Figure 7. Partition of energy of $\mathrm{qP}$ waves due to incident $\mathrm{qP}$ waves in rigid boundary. 


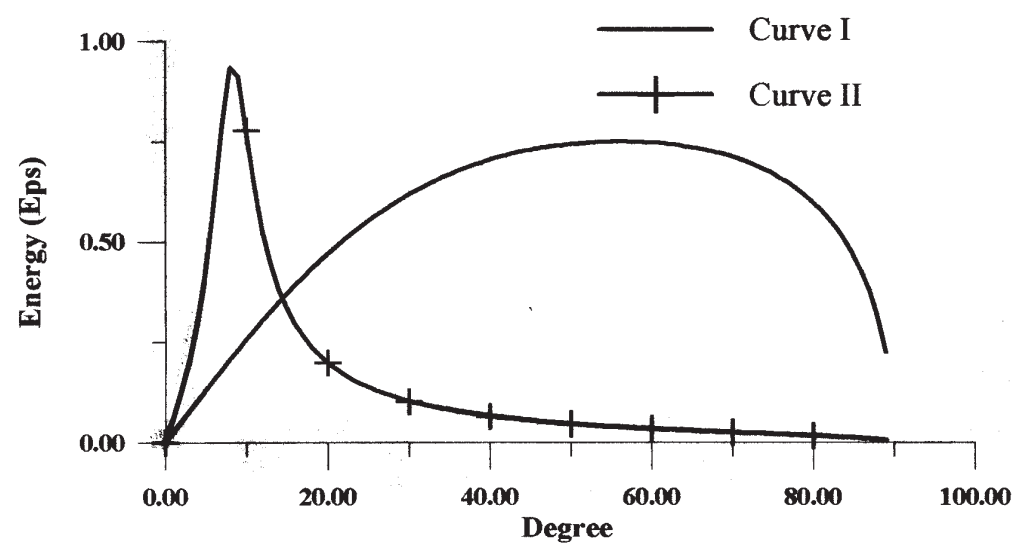

Figure 8. Partition of energy of qSV waves due to incident $\mathrm{qP}$ waves in rigid boundary.

for $A_{2} / A_{0}$ in fibre reinforced medium and compared with those in isotropic media (curve I). In this case, due to the effect of fibre-reinforced medium the values of $A_{2} / A_{0}$ are greater compared to the isotropic case.

This rigid boundary plays a very important role in case of reflection phenomena. All the values of $A_{1} / A_{0}$ (figure 13) in case of fibre-reinforced material in a rigid boundary are less compared to the isotropic case.

Figure 14 shows the reflection coefficient $A_{2} / A_{0}$ for qSV wave. The values of $A_{2} / A_{0}$ for fibre-reinforced media with rigid boundaries coincide with the values of $A_{2} / A_{0}$ for isotropic media at $32^{\circ}$. This is the critical point. The values for fibre-reinforced media (curve II) are less compared to those for isotropic media from $5^{\circ}$ to $31^{\circ}$.

Figures 11, 12, 15 and 16 show the partition of energy for incident qSV waves due to free and rigid boundaries.

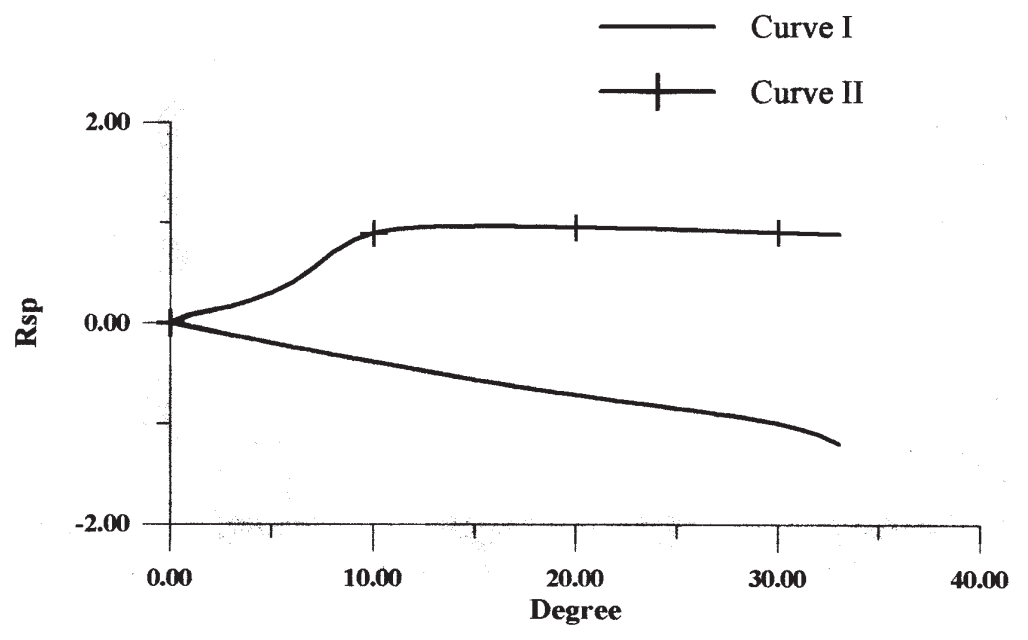

Figure 9. Amplitude ratios of $\mathrm{qP}$ waves due to incident $\mathrm{qSV}$ waves. 


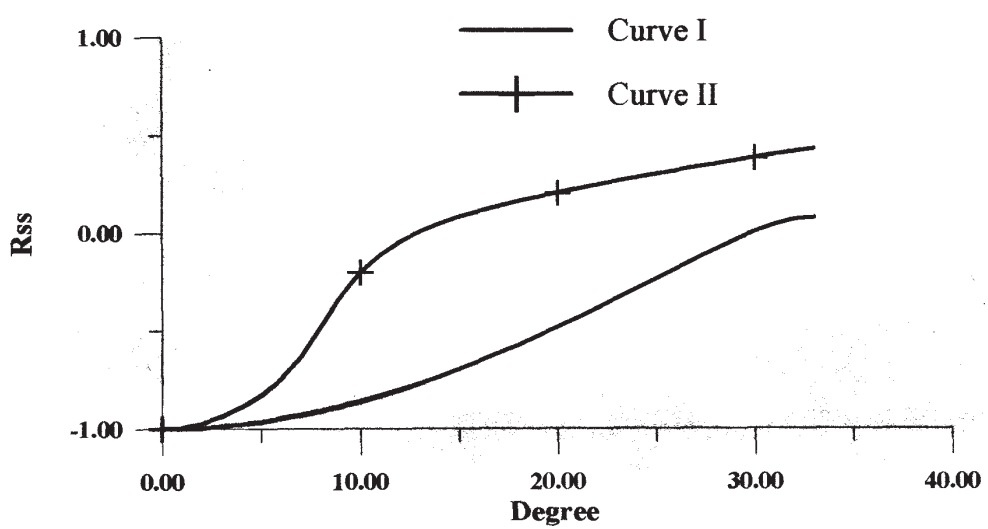

Figure 10. Amplitude ratios of qSV waves due to incident qSV waves.

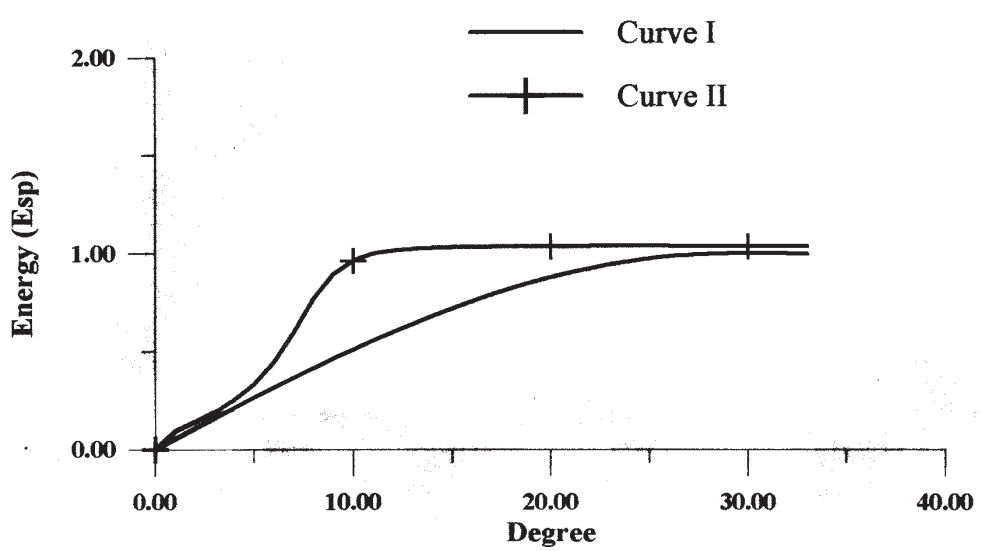

Figure 11. Partition of energy of $\mathrm{qP}$ waves due to incident $\mathrm{qSV}$ waves.

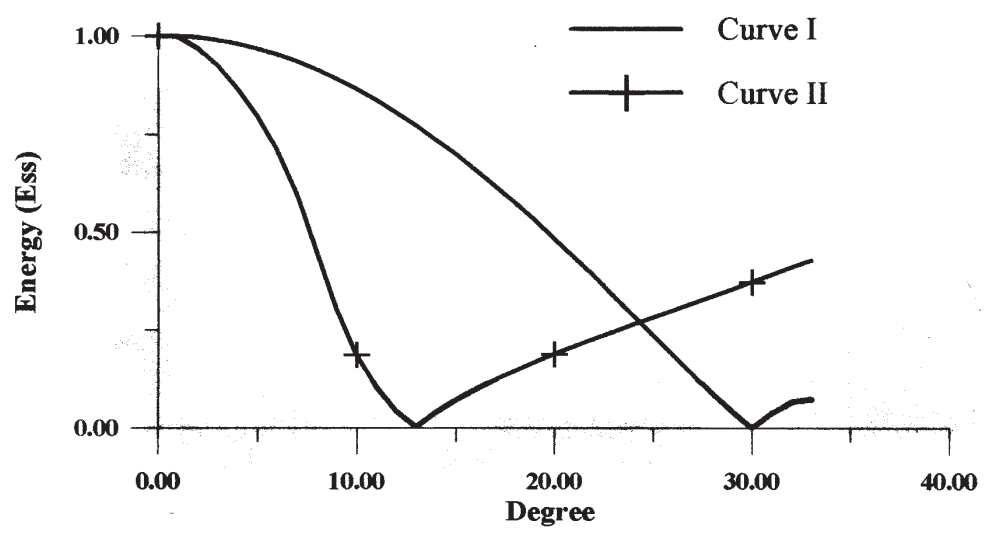

Figure 12. Partition of energy of qSV waves due to incident qSV waves. 


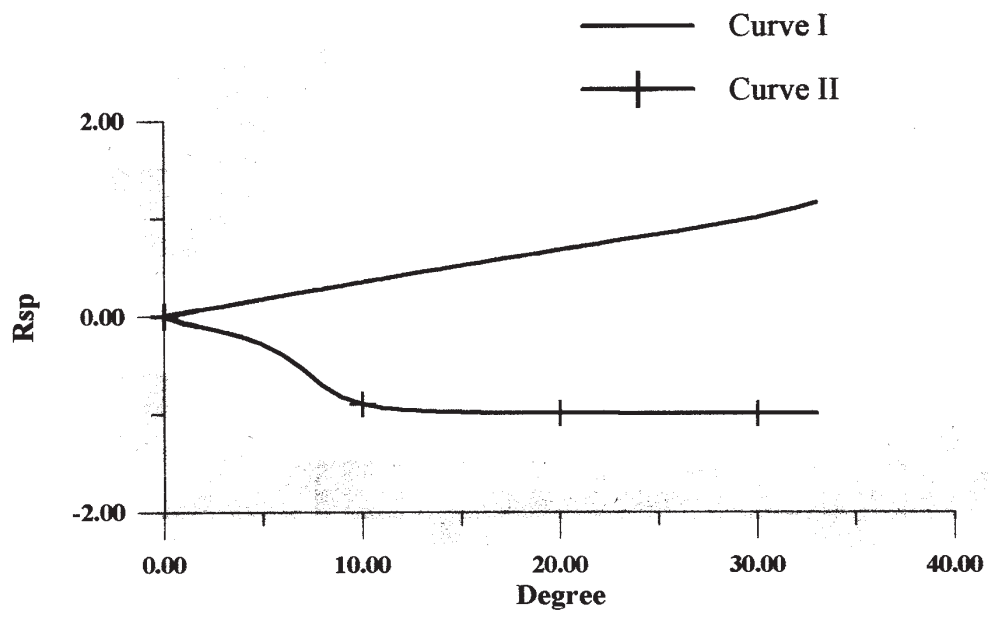

Figure 13. Amplitude ratios of $\mathrm{qP}$ waves due to incident $\mathrm{qSV}$ waves in rigid boundary.

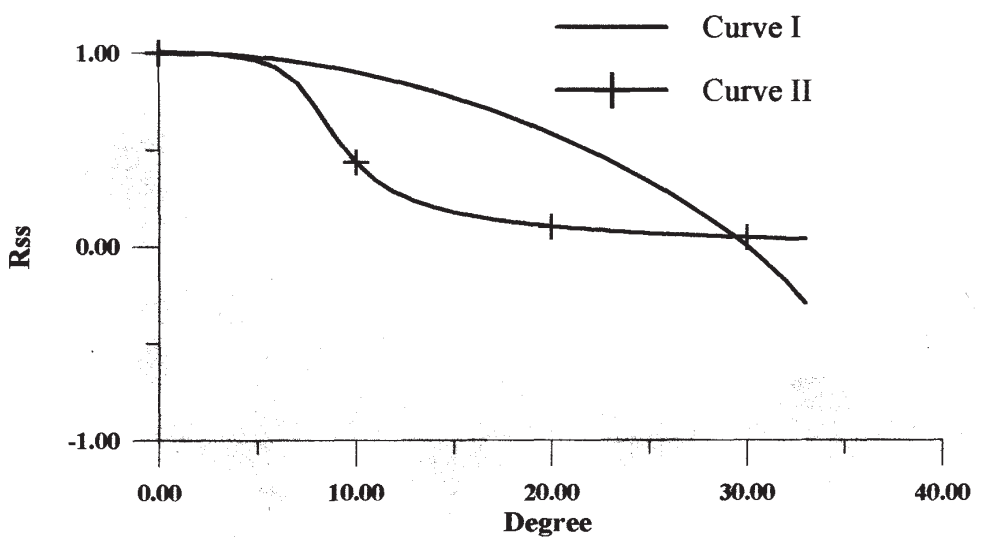

Figure 14. Amplitude ratios of qSV waves due to incident qSV waves in rigid boundary.

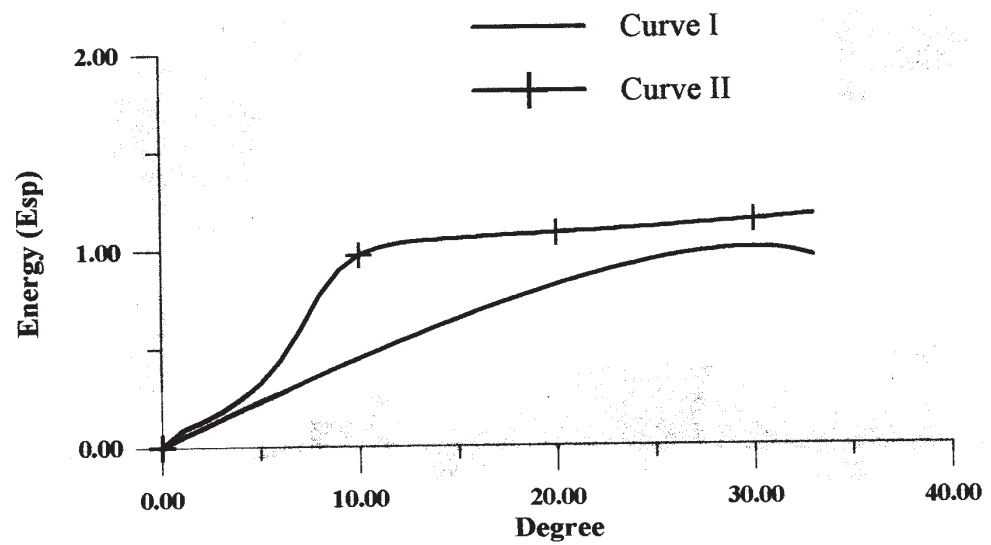

Figure 15. Partition of energy of $\mathrm{qP}$ waves due to incident $\mathrm{qSV}$ waves in rigid boundary. 


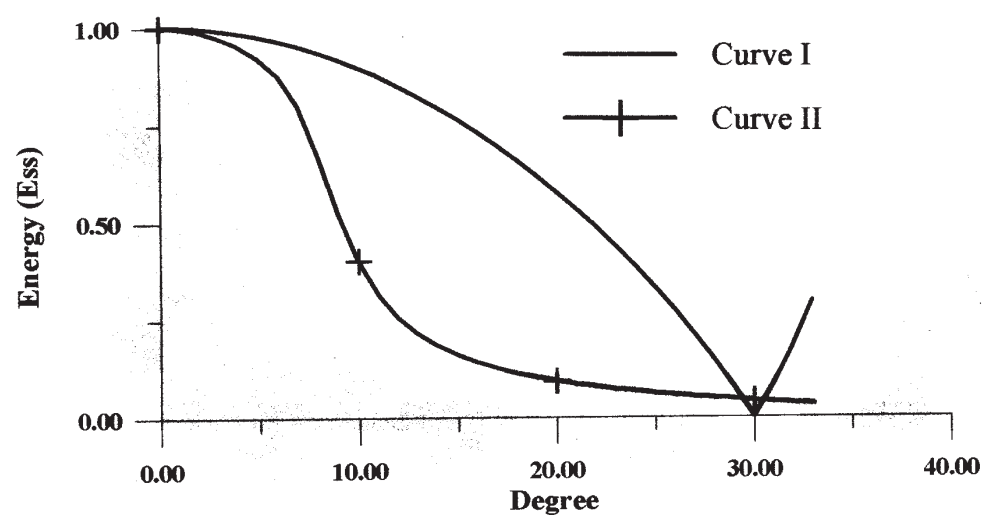

Figure 16. Partition of energy of qSV waves due to incident qSV waves in rigid boundary.

The authors are grateful to the referees for comments and valuable suggestions.

\section{References}

Achenbach J D 1976 Wave propagation in elastic solids (New York: North Holland)

Chattopadhyay A, Choudhury S 1995 The reflection phenomena of P-waves in a medium of monoclinic type. Int. J. Eng. Sci. 33: 195-207

Chattopadhyay A, Venkateswarlu R L K 1998 Stresses produced in fibre-reinforced half space due to a moving load. Bull. Cal. Math Soc. 90: 337-342

Chattopadhyay A, Saha S, Chakraborty M 1996 Reflection of SV waves in a monoclinic medium. Indian J. Pure Appl. Math. 27: 1029-1042

Crampin S 1975 Distinctive particle motion of surface waves as a diagnostic of anisotropic layering. Geophs. J.R. Astron. Soc. 40: 177-186

Crampin S, Taylor D B 1971 The propagation of surface waves in anisotropic media. Geophys. J.R. Astron. Soc. 25: 71-87

Dey S, Addy S K 1979 Reflection and refraction of plane waves under initial stresses at an interface. Int. J. Non-linear Mech. 14: 101-110

Gutenberg B 1944 Energy ratio of reflected and refracted seismic waves. Bull. Seismol. Soc. Am. 34: $85-112$

Henneke E G 1972 Reflection-refraction of stress waves at a plane boundary between anisotropic media. J. Acoust. Soc. Am. 51: 210-217

Keith C M, Crampin S 1977a Seismic body waves in anisotropic media, reflection and refraction at a plane interface. Geophys. J. R. Astron. Soc. 49: 181-208

Keith C M, Crampin S 1977b Seismic body waves in anisotropic media: propagation through a layer. Geophys. J. R. Astron. Soc. 49: 209-223

Keith C M, Crampin S 1977c Seismic body waves in anisotropic media, synthetic seismograms. Geophys. J. R. Astron. Soc. 49: 225-243

Knott C G 1899 Reflection and refraction of elastic waves with seismological applications. Philos. Mag. 48: 64-97

Markham MF 1970 Measurements of elastic constants of fibre composites by ultrasonics. Composites. 1: $145-149$

Norris A N 1983 Propagation of planes waves in a pre-stressed elastic medium. J. Accoust. Soc. Am. 74: $1642-1643$ 
Pal A K, Chattopadhyay A 1984 The reflection phenomena of plane waves at a free boundary in a prestressed elastic half-space. J. Accoust. Soc. Am. 76: 924-925

Richter C F 1958 Elementary seismology (San Francisco and London: W H Freeman)

*Singh S J, Khurana S 2002 Reflection of P and SV waves at the interface of a monoclinic elastic half-space. Proc. Indian Acad. Sci. (Earth Planet. Sci.) 111: 401-412

Spencer A J M 1972 Deformations of fibre-reinforced materials (London: Oxford University Press)

Thapliyal V 1974 Reflection of SH waves from anisotropic transition layer. Bull. Seismol. Soc. Am. 65: 1979-1991

Tolstoy I 1982 On elastic waves in pre-stressed solids. J. Geophy. Res. 87: 6823-6827

Udias A 1999 Principles of seismology (Cambridge: University Press)

${ }^{*}$ This reference added in proofs 